University of Wollongong

Research Online

Faculty of Engineering and Information

Faculty of Engineering and Information

Sciences - Papers: Part A

Sciences

$1-1-2010$

Iterative soft compensation for OFDM systems with clipping and superposition coded modulation

Jun Tong

University of Newcastle, jtong@uow.edu.au

Li Ping

City University of Hong Kong

Zhonghao Zhang

City University of Hong Kong

Vijay Bhargava

University of British Columbia

Follow this and additional works at: https://ro.uow.edu.au/eispapers

Part of the Engineering Commons, and the Science and Technology Studies Commons

Research Online is the open access institutional repository for the University of Wollongong. For further information contact the UOW Library: research-pubs@uow.edu.au 


\title{
Iterative soft compensation for OFDM systems with clipping and superposition coded modulation
}

\author{
Abstract \\ This paper deals with the clipping method used in orthogonal frequency-division multiplexing (OFDM) \\ systems to reduce the peak-to-average power ratio (PAPR). An iterative soft compensation method is \\ proposed to mitigate the clipping distortion, which can outperform conventional treatments. The impact \\ of signaling schemes on the residual clipping noise power is studied via the symbol variance analysis. It \\ is found that superposition coded modulation (SCM) can minimize the residual clipping noise power \\ among all possible signaling schemes. This indicates that SCM-based OFDM systems are more robust to \\ clipping effect than other alternatives when soft compensation is applied. It is also shown that a multi- \\ code SCM scheme can further reduce the clipping effect and its overall performance can be quickly \\ evaluated using a semi-analytical evolution method. Numerical examples are provided to verify the \\ analysis.

\section{Keywords} \\ coded, modulation, compensation, iterative, ofdm, soft, systems, clipping, superposition

\section{Disciplines} \\ Engineering | Science and Technology Studies

\section{Publication Details} \\ J. Tong, L. Ping, Z. Zhang \& V. K. Bhargava, "Iterative soft compensation for OFDM systems with clipping \\ and superposition coded modulation," IEEE Transactions on Communications, vol. 58, (10) pp. 2861-2870, \\ 2010.
}




\title{
Iterative Soft Compensation for OFDM Systems with Clipping and Superposition Coded Modulation
}

\author{
Jun Tong, Li Ping, Fellow, IEEE, Zhonghao Zhang, and Vijay K. Bhargava, Fellow, IEEE
}

\begin{abstract}
This paper deals with the clipping method used in orthogonal frequency-division multiplexing (OFDM) systems to reduce the peak-to-average power ratio (PAPR). An iterative soft compensation method is proposed to mitigate the clipping distortion, which can outperform conventional treatments. The impact of signaling schemes on the residual clipping noise power is studied via the symbol variance analysis. It is found that superposition coded modulation (SCM) can minimize the residual clipping noise power among all possible signaling schemes. This indicates that SCM-based OFDM systems are more robust to clipping effect than other alternatives when soft compensation is applied. It is also shown that a multi-code SCM scheme can further reduce the clipping effect and its overall performance can be quickly evaluated using a semi-analytical evolution method. Numerical examples are provided to verify the analysis.
\end{abstract}

Index Terms-Clipping, iterative decoding, orthogonal frequency-division multiplexing (OFDM), soft compensation, superposition coded modulation (SCM).

\section{INTRODUCTION}

$\mathbf{O}$ RTHOGONAL frequency-division multiplexing (OFDM) is a multi-carrier transmission technique for broadband channels. It has attracted tremendous attention due to its high spectral efficiency and low receiver complexity. However, OFDM systems suffer from a high peak-to-average power ratio (PAPR) problem. This makes OFDM more prone to the non-linear effect of transmitter devices than single-carrier schemes.

Various PAPR reduction techniques have been investigated (see [1] and the references therein). They can be broadly classified into two categories. The first category involves redundancy (and so spectral efficiency loss), such as coding, partial transmit sequence, and selective mapping techniques. The second category does not involve redundancy, such as deliberate clipping [2]-[18].

Clipping may cause non-linear distortion which degrades performance. To treat such distortion, iterative techniques have

Paper approved by E. Perrins, the Editor for Modulation Theory of the IEEE Communications Society. Manuscript received June 9, 2009; revised February 12, 2010.

J. Tong was with the Department of Electronic Engineering, City University of Hong Kong, and is now with the School of Electrical Engineering and Computer Science, The University of Newcastle, NSW, Australia (e-mail: eejtong@gmail.com).

L. Ping and Z. Zhang are with the Department of Electronic Engineering, City University of Hong Kong, Hong Kong SAR, China (e-mail: eeliping@cityu.edu.hk, zhozhang@student.cityu.edu.hk).

V. K. Bhargava is with the Department of Electrical and Computer Engineering, The University of British Columbia, Vancouver BC, Canada.

This work was supported by a grant from the Research Grant Council of the Hong Kong Special Administrative Region, China, under project CityU 116706. This paper was presented in part at IEEE GlobeCom 2008, New Orleans, USA.

Digital Object Identifier 10.1109/TCOMM.2010.083110.09296 been studied. In [12]-[15], clipping distortion is estimated and then partially canceled at the receiver. A key step in the works in [12]-[15] is to compute the means for the original signals before clipping using decoder feedbacks. The technique developed in [28] involves both means and variances of the original signals, which offers noticeable performance gains. The focus of [28] is on superposition coded modulation (SCM) in single-carrier environments and the discussion on OFDM systems is very limited.

This paper is concerned with the treatment of clipping distortion for OFDM systems with coded modulation. The proposed technique combines the works in [12]-[15] and [28]: Price's result is used to remove the correlation between the original signals and clipping distortions; and both means and variances of the original signals computed from the decoder feedbacks are used to estimate the clipping distortions. This combined technique ensures the effectiveness of iterative detection and brings about considerable performance gains.

As discussed in [28], SCM generally exhibits high PAPR. However, since OFDM suffers from high PAPR anyway, the use of SCM in OFDM does not worsen the problem. On the contrary, based on a result from [41], we will show that SCM is advantageous (compared with other alternative coded modulation methods) for estimating the original signals. This advantage implies that SCM-OFDM systems may lead to better performance with a low clipping ratio and hence can help reduce the PAPR. Moreover, further performance improvement can be achieved using a multi-code SCM scheme. These properties are confirmed by simulation results.

We also develop a fast, semi-analytical technique to predict the performance of an iterative receiver. The presence of several different types of distortions, namely, additive white Gaussian noise (AWGN), frequency-selective fading, interlayer interference (due to SCM) and clipping distortion, makes the problem complicated. We will develop a procedure to handle such difficulty. It involves (i) the approximation of the clipping distortions plus inter-layer interferences using additive Gaussian variables and (ii) a two-dimensional precalculated table to separate the different types of distortions. The resultant prediction method is reasonably accurate. The availability of a fast analysis technique provides a useful tool for searching-based system design.

Combining the above mentioned techniques, we obtain a promising solution to the PAPR problem in OFDM systems. For example, we show that performance within $1.35 \mathrm{~dB}$ away from the Shannon limit can be achieved with a severe clipping ratio $(\mathrm{CR})$ as low as $0 \mathrm{~dB}$.

The rest of this paper is organized as follows. Section II 
introduces the system model and iterative soft compensation method. The impact of signaling schemes on the residual clipping noise power and SCM schemes are discussed in Section III. Numerical results are presented in Section IV, followed by the conclusion in Section V.

\section{ITERATIVE SOFT COMPENSATION}

\section{A. System Model}

Consider a coded OFDM system illustrated in Fig. 1. At the transmitter, the information bits are first encoded by a binary encoder (ENC). The resultant coded bits are then randomly interleaved and packed into groups $\{\boldsymbol{b}[n]=$ $\left.\left(b_{1}[n], b_{2}[n], \cdots, b_{K}[n]\right)\right\}$ of $K$ bits. Each $\boldsymbol{b}[n]$ is mapped to a symbol $X[n]$ to be carried by the $n$th sub-carrier. (The related mapping rule will be detailed in Section III-A.)

Let $N$ be the number of sub-carriers and define $\boldsymbol{X}=$ $[X[0], X[1], \cdots, X[N-1]]^{\mathrm{T}}$. Then $\{X[n]\}$ are modulated onto sub-carriers using the inverse discrete Fourier transform (IDFT). The resultant signal is over-sampled into $\boldsymbol{x}=$ $[x[0], x[1], \cdots, x[L N-1]]^{\mathrm{T}}$ with over-sampling factor $L$,

$$
\boldsymbol{x}=\boldsymbol{F}^{\dagger} \boldsymbol{X},
$$

where $\boldsymbol{F}$ is an $N \times L N$ matrix with $(n, m)$ th entry given by $e^{-j \frac{2 \pi m n}{L N}} / \sqrt{N L}, j=\sqrt{-1}$, and "(.) ${ }^{\dagger}$ " denotes conjugate transpose of matrix. To reduce the PAPR, each entry $x[m]$ of $\boldsymbol{x}$ is deliberately clipped using the following clipping function,

$$
g(x[m])=\left\{\begin{array}{ccc}
x[m], & \text { if } & |x[m]| \leq A \\
A x[m] /|x[m]|, & \text { if } & |x[m]|>A
\end{array}\right.
$$

where $A$ is a clipping threshold and $|\cdot|$ represents absolute value. The clipping ratio in decibel is defined as $\mathrm{CR}=$ $10 \log _{10}\left(A^{2} / \mathrm{E}\left[|x[m]|^{2}\right]\right)$ where $\mathrm{E}[\cdot]$ represents mathematical expectation. With abuse of notation, we write the clipped signal vector as $g(\boldsymbol{x})$.

Clipping introduces in-band non-linear distortion and outof-band radiation [3], [6]. This paper focuses only on the inband distortion that degrades the bit-error-rate (BER) performance [2]-[18]. We assume that ideal bandpass filtering [3] is applied to $g(\boldsymbol{x})$ and the out-of-band radiation is perfectly mitigated. Cyclic prefix is then added to the filtered signal to treat inter-block-interference (IBI) and the resultant signal is transmitted. For simplicity, we assume that

- clipping is the only source of non-linear distortion, which implies that linear power amplifiers are used;

- the cyclic prefix is sufficiently long so that the IBI can be perfectly removed; and

- the channel coefficients remain unchanged within each OFDM block and are perfectly known at the receiver.

Denote by $\boldsymbol{y}$ the time-domain received signal vector. After removing the cyclic prefix and applying the discrete Fourier transform (DFT) to $\boldsymbol{y}$, we obtain a frequency-domain signal vector $\boldsymbol{Y}=[Y[0], Y[1], \cdots, Y[N-1]]^{\mathrm{T}}$. Considering (1) and (2), we model $\boldsymbol{Y}$ as

$$
\boldsymbol{Y}=\boldsymbol{H} \boldsymbol{F} g(\boldsymbol{x})+\boldsymbol{W}
$$

where $\boldsymbol{H}=\operatorname{diag}(H[0], H[1], \cdots, H[N-1])$ consists of the fading coefficients on the $N$ subcarriers and $\boldsymbol{W}$ is a vector of samples of a complex Gaussian noise with mean zero and variance $\sigma_{W}^{2} / 2$ per dimension.

The suboptimal receiver consists of an elementary signal estimator (ESE) and a decoder (DEC) connected by an interleaver $(\Pi)$ and a de-interleaver $\left(\Pi^{-1}\right)$. They operate iteratively following the principles of bit-interleaved coded modulation with iterative decoding (BICM-ID) [19], [20].

\section{B. Iterative Detection/Decoding}

The clipping effect is handled by the soft compensation (SC) module in Fig. 1. Applying Price's theorem for Gaussianinput memoryless non-linear systems [32], the clipped signal vector $g(\boldsymbol{x})$ is approximated as

$$
g(\boldsymbol{x})=\alpha \boldsymbol{x}+\boldsymbol{d}
$$

where $\boldsymbol{d}=[d[0], \cdots, d[L N-1]]^{\mathrm{T}}$ and $\alpha$ is a constant scalar computed as

$$
\alpha=\frac{\mathrm{E}\left[\boldsymbol{x}^{\dagger} g(\boldsymbol{x})\right]}{\mathrm{E}\left[\|\boldsymbol{x}\|^{2}\right]}
$$

and $\|\cdot\|$ denotes the Frobenius norm of a vector. Then from (3) and $\boldsymbol{F} \boldsymbol{F}^{\dagger}=\boldsymbol{I}$, we have

$$
\boldsymbol{Y}=\alpha \boldsymbol{H} \boldsymbol{X}+\boldsymbol{H} \boldsymbol{F d}+\boldsymbol{W} .
$$

Assume that the mean of $\boldsymbol{d}$, denoted by $\overline{\boldsymbol{d}}$ below, is available. (At the beginning of decoding, a common choice is $\overline{\boldsymbol{d}}=0$. The details in updating $\overline{\boldsymbol{d}}$ will be given in (12)). To reduce the distortion related to $\boldsymbol{d}$, we subtract $\boldsymbol{H} \boldsymbol{F} \overline{\boldsymbol{d}}$ from $\boldsymbol{Y}$, yielding

$$
\boldsymbol{Z}=\boldsymbol{Y}-\boldsymbol{H} \boldsymbol{F} \overline{\boldsymbol{d}}=\alpha \boldsymbol{H} \boldsymbol{X}+\boldsymbol{\Xi}
$$

where

$$
\boldsymbol{\Xi}=\boldsymbol{H} \boldsymbol{F}(\boldsymbol{d}-\overline{\boldsymbol{d}})+\boldsymbol{W}
$$

is the residual clipping noise plus channel noise. Clearly, $\boldsymbol{d}-\overline{\boldsymbol{d}}$ has zero mean, and so does $\boldsymbol{F}(\boldsymbol{d}-\overline{\boldsymbol{d}})$.

Assumption 1: The entries in $\boldsymbol{F}(\boldsymbol{d}-\overline{\boldsymbol{d}})$ are independent, identically distributed (i.i.d.) Gaussian random variables that are uncorrelated with $\boldsymbol{x}$.

This assumption can be justified as follows.

1) Following general treatments, the entries of $\boldsymbol{x}$ are approximately uncorrelated. Then the entries of $\boldsymbol{d}-\overline{\boldsymbol{d}}$ are approximately uncorrelated, so are the entries of $\boldsymbol{F}(\boldsymbol{d}-\overline{\boldsymbol{d}})$ since $\boldsymbol{F}$ is orthogonal. According to the central limit theorem, the entries of $\boldsymbol{F}(\boldsymbol{d}-\overline{\boldsymbol{d}})$ are approximately Gaussian since they are weighted sums of many uncorrelated random variables.

2) Due to the use of $\alpha$ in (4), $\boldsymbol{d}-\overline{\boldsymbol{d}}$ and $\boldsymbol{x}$ are uncorrelated, i.e., $\mathrm{E}\left[(\boldsymbol{d}-\overline{\boldsymbol{d}}) \boldsymbol{x}^{\dagger}\right]=\mathbf{0}$, so are $\boldsymbol{F}(\boldsymbol{d}-\overline{\boldsymbol{d}})$ and $\boldsymbol{x}$.

Let $\{\mathrm{V}[d[n]]\}$ be the variance of the entries in $\boldsymbol{d}$. Since the entries in $\boldsymbol{F}$ have the same absolute value of $1 / \sqrt{L N}$, the entries in $\boldsymbol{F}(\boldsymbol{d}-\overline{\boldsymbol{d}})$ have the same variance denoted by $v_{d}$ where

$$
v_{d}=\frac{1}{L N} \sum_{m=0}^{L N-1} \mathrm{~V}[d[m]] .
$$

We will consider the details in evaluating $v_{d}$ in Section II-C. From Assumption 1 and (9), we can make the following assumption. 


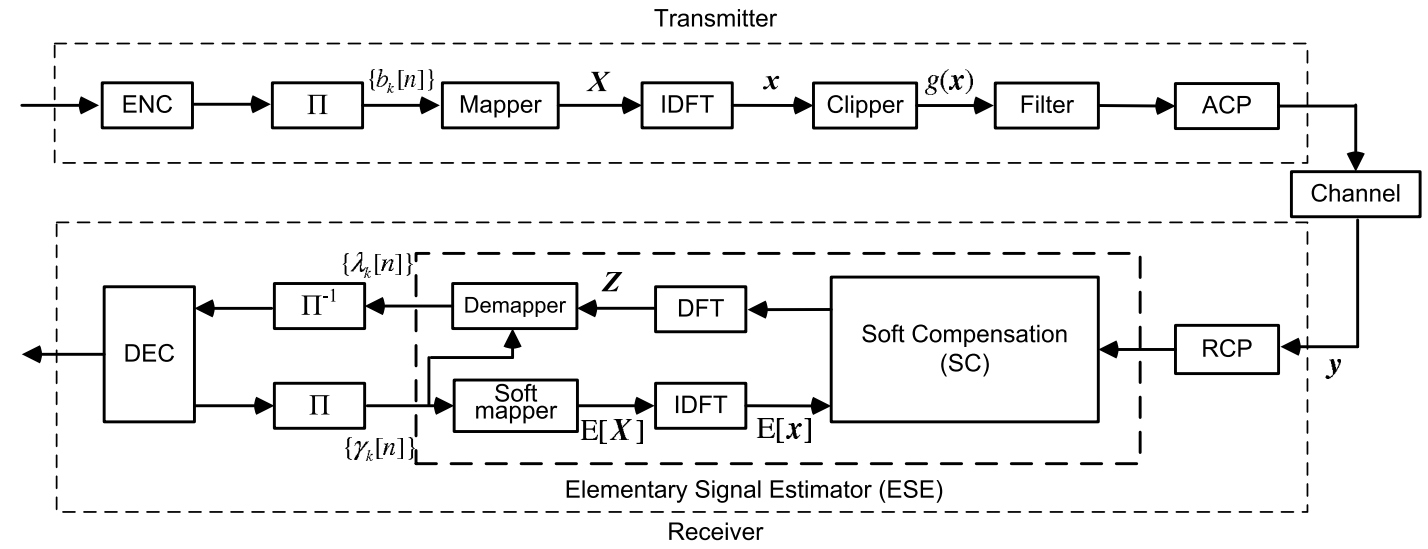

Fig. 1. Coded OFDM systems with clipping, where $\Pi$ denotes interleaver and $\Pi^{-1}$ de-interleaver. ACP denotes adding cyclic prefix and RCP removing cyclic prefix.

Assumption 2: $\Xi=\{\Xi[n]\}$ are independent Gaussian random variables with mean $\mathrm{E}[\Xi[n]]=0$ and variance $\mathrm{V}[\Xi[n]]=|H[n]|^{2} v_{d}+\sigma_{W}^{2}$.

Now return to the detection problem based on (7) and (8). From Assumption 1, the residual clipping noise $\boldsymbol{F}(\boldsymbol{d}-\overline{\boldsymbol{d}})$ is uncorrelated with $\boldsymbol{X}$ and symbol-by-symbol detection can be applied. The soft demapper outputs the so-called extrinsic log-likelihood ratios (LLRs):

$$
\begin{gathered}
\lambda_{k}[n]=\ln \left(\frac{\operatorname{Pr}\left(b_{k}[n]=0 \mid Z[n],\left\{\gamma_{k}[n]\right\}\right)}{\operatorname{Pr}\left(b_{k}[n]=1 \mid Z[n],\left\{\gamma_{k}[n]\right\}\right)}\right)-\gamma_{k}[n], \\
k=1,2, \cdots, K, n=0,1, \cdots, N-1,
\end{gathered}
$$

where $Z[n]$ denotes the $n$th entry of $\boldsymbol{Z}$, and $\gamma_{k}[n]$ is the a priori LLR about $b_{k}[n]$ which can be obtained as the extrinsic LLR from the DEC (see (11)). In general cases, the a posteriori probability (APP) demapping [19], [33] can be used to evaluate (10). For SCM, more details regarding evaluating (10) will be discussed in Section III.

The DEC takes the de-interleaved version of $\left\{\lambda_{k}[n]\right\}$ in (10) as inputs and performs standard APP decoding. The extrinsic LLRs are produced by the DEC based on coding constraint $\mathcal{C}$ :

$$
\begin{gathered}
\gamma_{k}[n]=\ln \left(\frac{\operatorname{Pr}\left(b_{k}[n]=0 \mid \mathcal{C},\left\{\lambda_{k}[n]\right\}\right)}{\operatorname{Pr}\left(b_{k}[n]=1 \mid \mathcal{C},\left\{\lambda_{k}[n]\right\}\right)}\right)-\lambda_{k}[n], \\
k=1,2, \cdots, K, n=0,1, \cdots, N-1 .
\end{gathered}
$$

The DEC feedback $\left\{\gamma_{k}[n]\right\}$ will be then used to update $\overline{\boldsymbol{d}}$ in an iterative manner, as detailed in the next subsection.

\section{Proposed Clipping Noise Estimation Method}

The following assumption is the key to the soft compensation method proposed in this paper.

Assumption 3: Each entry $x$ in $\boldsymbol{x}$ is a Gaussian random variable.

This assumption can be justified by the central limit theorem since $x$ is a weighted sum of $N$ independent random variables $\{X[n]\}$. It also leads to a low-cost method to compensate the distortion caused by $\boldsymbol{d}$ at the receiver.

For simplicity, we assume that the real and imaginary parts of $x$ have the same variance $\mathrm{V}[x] / 2$, where $\mathrm{V}[x]=\mathrm{E}[\mid x-$ $\left.\left.\mathrm{E}[x]\right|^{2}\right]$ is referred to as the symbol variance. The mean $\mathrm{E}[x]$ and variance $\mathrm{V}[x]$ can be estimated from $\left\{\gamma_{k}[n]\right\}$, as will be detailed in Section III-A. With the Gaussian approximation, the distribution of $x$ is fully characterized by $\mathrm{E}[x]$ and $\mathrm{V}[x]$. According to [31], given a priori information about $x$, the optimal estimate of $d=g(x)-\alpha x$ (in terms of minimization of the mean square error (MSE)) is the conditional mean of $d$. Therefore, $\bar{d}$ (an entry in $\overline{\boldsymbol{d}}$ ) can be computed as

$$
\begin{aligned}
\bar{d} & =\mathrm{E}[g(x)-\alpha x] \\
& =\int \frac{g(x)-\alpha x}{\pi \mathrm{V}[x]} \exp \left(-\frac{|x-\mathrm{E}[x]|^{2}}{\mathrm{~V}[x]}\right) d x .
\end{aligned}
$$

The corresponding residual clipping noise power can be estimated by

$$
\begin{aligned}
\mathrm{V}[d] & =\mathrm{E}\left[|d-\bar{d}|^{2}\right] \\
& =\int \frac{|g(x)-\alpha x-\bar{d}|^{2}}{\pi \mathrm{V}[x]} \exp \left(-\frac{|x-\mathrm{E}[x]|^{2}}{\mathrm{~V}[x]}\right) d x,
\end{aligned}
$$

which can be used to generate the variance of the entries of $\boldsymbol{\Xi}$ in (8). In practice, (12) and (13) can be tabulated as functions of $(\mathrm{E}[x], \mathrm{V}[x])$ for online evaluation, following [28], which involves two two-dimensional tables.

Note that in the proposed soft compensation method, we make no assumption regarding the distribution of $\boldsymbol{d}$. The reason is that, under Assumption $1, \boldsymbol{F}(\boldsymbol{d}-\overline{\boldsymbol{d}})$ contains uncorrelated, Gaussian entries, and so its distribution can be fully characterized by the means and variances in (12) and (13).

Based on the above discussions, we list the clipping noise estimation procedure as follows.

(i) Estimate the mean $\mathrm{E}[X[n]]$ and variance $\mathrm{V}[X[n]]$ of $X[n]$ (the $n$th entry of $\boldsymbol{X}$ ) from $\left\{\gamma_{k}[n]\right\}$, as detailed in Section III-A.

(ii) Generate the means and variances of the entries of $\boldsymbol{x}$ based on the relationship $\boldsymbol{x}=\boldsymbol{F}^{\dagger} \boldsymbol{X}$. More specifically, $\mathrm{E}[\boldsymbol{x}]=\boldsymbol{F}^{\dagger} \mathrm{E}[\boldsymbol{X}]$ where $\mathrm{E}[\boldsymbol{x}]$ and $\mathrm{E}[\boldsymbol{X}]$ are respectively the means of $\boldsymbol{x}$ and $\boldsymbol{X}$. The variance of $x[m], \forall m$, is computed as $\mathrm{V}[x[m]]=1 /(L N) \sum_{n=0}^{N-1} \mathrm{~V}[X[n]]$.

(iii) Generate the means and variances of the entries of $\boldsymbol{d}$ using (12) and (13). In this way, the variance of the entries of $\boldsymbol{F}(\boldsymbol{d}-\overline{\boldsymbol{d}})$ in (8) is computed as $v_{d}=$ $1 /(L N) \sum_{m=0}^{L N-1} \mathrm{~V}[d[m]]$. 
Steps (i)-(iii) are performed respectively by the soft mapper, IDFT, and SC modules in the ESE. The ESE/DEC operations outlined above can be repeated iteratively.

\section{Comparisons with Existing Methods}

The following methods have been proposed in [3], [12]-[15] and [28] to treat the clipping effect.

- In [3], the clipping noise is approximated by a zero-mean Gaussian noise independent of the wanted signal. The variance of the clipping noise is estimated and the impact of clipping noise is treated together with channel noise. Decoder feedbacks are not required for this purpose.

- The clipping noise is estimated as $\overline{\boldsymbol{d}}=g(\mathrm{E}[\boldsymbol{x}])-\alpha \mathrm{E}[\boldsymbol{x}]$ in [12]-[15]. The variance of $\boldsymbol{x}$ is not required in this approach.

- The method in [28] involves both mean and variance of $\boldsymbol{x}$, which makes better use of the decoder feedback. The focus of [28] is plain SCM and OFDM is only briefly mentioned in [28]. The clipping operation is modeled as $g(\boldsymbol{x})=\boldsymbol{x}+\boldsymbol{d}$ in [28] (without $\alpha$ in (4)) where $\boldsymbol{d}$ is correlated with $\boldsymbol{x}$. The correlation between $\boldsymbol{d}$ and $\boldsymbol{x}$ complicates the detection of $\boldsymbol{x}$. A hypothesisdependant technique is devised in [28, Section IV-G] to handle this correlation problem. When applied to OFDM systems, however, this technique incurs excessively high complexity. A simplified method that ignores correlation is used for OFDM systems in [28] to save complexity, which, unfortunately, leads to noticeable performance degradation, as shown in Fig. 7 below.

The scheme outlined above combines the techniques in [12][15] and [28]. Removing the correlation between $\boldsymbol{d}$ and $\boldsymbol{x}$ means that $\boldsymbol{d}$ can be more accurately modeled as additive noise samples, which has two beneficial consequences. First, the estimation of $\bar{d}$ and $\mathrm{V}[d]$ based on (12) and (13) provides a much simpler solution than the hypothesis-dependent technique used in [28]. Second, we can apply a SNR evolution technique to analyze the performance of the iterative detection process and search for optimized system design, as will be discussed in Section III-C below.

Also, (approximate) maximum likelihood sequence detection is considered in [17] and [18] for uncoded OFDM systems involving clipping. This technique provides near-optimal performance in uncoded systems. However, it is difficult to apply this technique to coded cases due to excessively high complexity involved.

\section{E. Effect of the Uncertainty on Clipping Distortion}

We now analyze the effectiveness of the soft compensation technique in (7) and (8).

From Section II-C, the soft estimate $\bar{d}$ is computed from the DEC feedback $\left\{\gamma_{k}[n]\right\}$. Assumption 4 below follows the extrinsic information transfer (EXIT) chart principle [34], which is approximately true with sufficiently long random interleavers.

Assumption 4: $\left\{\gamma_{k}[n]\right\}$ are i.i.d. random variables.

Define $\mathbb{E}_{\gamma}[\cdot]$ the expectation with respect to the distribution of $\left\{\gamma_{k}[n]\right\}$, and $\sigma_{d}^{2}$ the average residual clipping noise power

$$
\sigma_{d}^{2} \equiv \mathbb{E}_{\gamma}\left[\|\boldsymbol{F}(\boldsymbol{d}-\overline{\boldsymbol{d}})\|^{2}\right] / N=\mathbb{E}_{\gamma}\left[\|\boldsymbol{d}-\overline{\boldsymbol{d}}\|^{2}\right] /(L N) .
$$

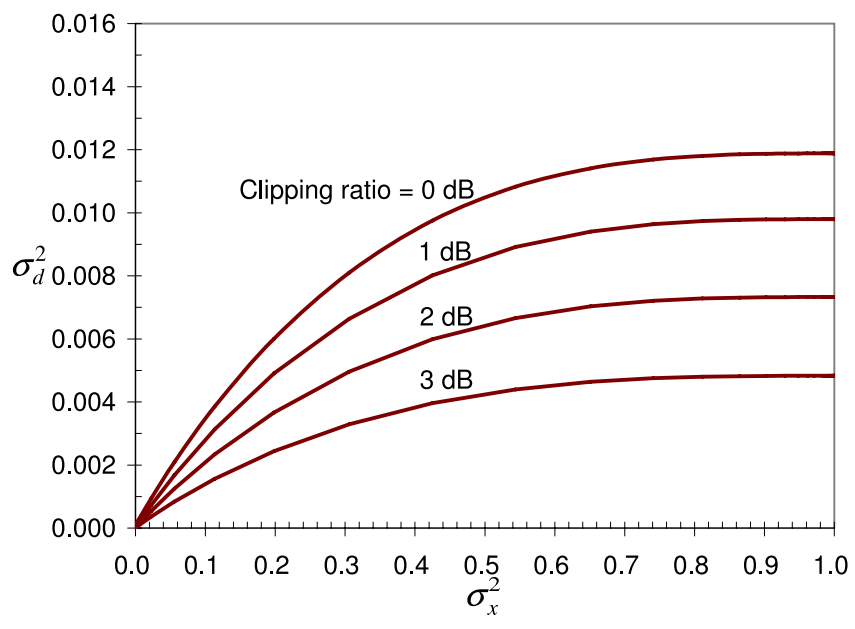

Fig. 2. $\sigma_{d}^{2}$ versus $\sigma_{x}^{2}$. The oversampling factor $L=4$. The number of sub-carriers $N=256$. The average signal power $\mathrm{E}\left[|X[n]|^{2}\right]$ is normalized to 1 .

Here, $\sigma_{d}^{2}$ represents the uncertainty on clipping distortion. If $\sigma_{d}^{2}=0$, i.e., $\boldsymbol{d}=\overline{\boldsymbol{d}}$, then the clipping distortion is completely removed from the received signal using (7). Thus we can use $\sigma_{d}^{2}$ as a measure for the effectiveness of the compensation operation in (7).

With interleaving, we assume that $\{X[n]\}$ are i.i.d.. Define symbol variance as

$$
\begin{aligned}
\sigma_{x}^{2} & \equiv \mathbb{E}_{\gamma}[\mathrm{V}[X[n]]]=\mathbb{E}_{\gamma}\left[\|\boldsymbol{X}-\mathrm{E}[\boldsymbol{X}]\|^{2}\right] / N \\
& =\mathbb{E}_{\gamma}\left[\|\boldsymbol{x}-\mathrm{E}[\boldsymbol{x}]\|^{2}\right] / N .
\end{aligned}
$$

The last equation in (15) holds since $\boldsymbol{x}=\boldsymbol{F}^{\dagger} \boldsymbol{X}, \mathrm{E}[\boldsymbol{x}]=$ $\boldsymbol{F}^{\dagger} \mathrm{E}[\boldsymbol{X}]$ and $\boldsymbol{F} \boldsymbol{F}^{\dagger}=\boldsymbol{I}$. Clearly, $\sigma_{x}^{2}$ represents the uncertainty about $\boldsymbol{x}$. From (12) and (13), the estimation of $\boldsymbol{d}$ is obtained based on that for $\boldsymbol{x}$. Therefore, $\sigma_{d}^{2}$ is an implicit function of $\sigma_{x}^{2}$ :

$$
\sigma_{d}^{2}=\phi\left(\sigma_{x}^{2}\right)
$$

Fig. 2 shows the $\sigma_{d}^{2}$ versus $\sigma_{x}^{2}$ curves (obtained by the Monte Carlo method) for different clipping ratios. We can observe that $\sigma_{d}^{2}$ is a monotonically increasing function of $\sigma_{x}^{2}$ but we are not able to rigorously prove this monotonicity yet. This observation is well within expectation: a more accurate estimate of $\boldsymbol{x}$ would lead to a more accurate estimate of $\boldsymbol{d}$. We have also observed in our simulation that $\phi(\cdot)$ defined in (16) is nearly independent of the signaling schemes employed for generating $\boldsymbol{X}$ from the coded bits $\left\{b_{k}[n]\right\}$. This is because when $N$ is sufficiently large, from the central limit theorem, the IDFT output $\boldsymbol{x}$ is always approximately Gaussian-distributed, regardless of the signaling schemes. As a consequence of these two observations, minimizing $\sigma_{d}^{2}$ is equivalent to minimizing $\sigma_{x}^{2}$.

In the next section, we will show that $\sigma_{x}^{2}$ and so $\sigma_{d}^{2}$ can be minimized using properly designed signaling schemes.

\section{Superposition Coded Modulation (SCM)}

In this section, we discuss the impact of signaling scheme on the performance of the iterative soft compensation method and show the optimality of the SCM signaling. We also show the advantage of multi-code SCM and analyze its performance. 


\section{A. Optimal Signaling Scheme for Soft Compensation}

Let $\mathcal{S}$ be a constellation of $2^{K}$ points. Let $b=$ $\left(b_{1}, b_{2}, \cdots, b_{K}\right)$ be a binary $K$-tuple with $b_{k} \in\{0,1\}$ and $\mathcal{B}$ the set of $2^{K}$ such $K$-tuples. A signaling scheme is defined by $(\mathcal{S}, \mathcal{R})$, where $\mathcal{R}$ is the mapping from $\mathcal{B}$ to $\mathcal{S}$. Some examples of $(\mathcal{S}, \mathcal{R})$ can be found in [19]-[23]. Another example is the SCM [24]-[30] that generates $X$ as a superposition of $K$ bipolar modulated signals,

$$
X=\sum_{k=1}^{K} \beta_{k}(-1)^{b_{k}},
$$

where the weighting factors $\left\{\beta_{k}\right\}$ are complex constants. We will refer to the operation in (17) as "SCM signaling". We can apply the SCM signaling to the transmitter in Fig. 1, which will result in a special case of BICM-ID [27].

Following the turbo principle, $\left\{b_{k}\right\}$ can be treated as binary random variables and so the mapped symbol $X$ is also random. Suppose a set of a priori LLRs $\left\{\gamma_{k}\right\}$ about $\left\{b_{k}\right\}$ are available.

$$
\gamma_{k} \equiv \ln \left(\frac{\operatorname{Pr}\left(b_{k}=0\right)}{\operatorname{Pr}\left(b_{k}=1\right)}\right), k=1,2, \cdots, K \text {. }
$$

(In the soft mapper, $\left\{\gamma_{k}\right\}$ are the feedback LLRs from the DEC.) Then the a priori probability for each $b_{k}$ can be computed as $\operatorname{Pr}\left(b_{k}=0\right)=1-\operatorname{Pr}\left(b_{k}=1\right)=$ $\frac{e^{\gamma_{k}}}{1+e^{\gamma_{k}}}, k=1,2, \cdots, K$. Let $\left\{s_{0}, s_{1}, \cdots, s_{2^{K}-1}\right\}$ be the set of the signaling points in $\mathcal{S}$. Now the a priori probability that $s_{m} \in \mathcal{S}$ is the transmitted symbol can be computed as $\operatorname{Pr}\left(X=s_{m}\right)=\prod_{k=1}^{K} \operatorname{Pr}\left(b_{k}\right)$, where $\operatorname{Pr}\left(b_{k}\right)$ is either $\operatorname{Pr}\left(b_{k}=0\right)$ or $\operatorname{Pr}\left(b_{k}=1\right)$, depending on $\mathcal{R}$. (We have assumed that $\left\{b_{1}, b_{2}, \cdots, b_{K}\right\}$ are independent.) Finally, the mean and variance of $X$ are, respectively,

$$
\begin{aligned}
& \mathrm{E}[X]=\sum_{m=0}^{2^{K-1}} s_{m} \operatorname{Pr}\left(X=s_{m}\right) \\
& \mathrm{V}[X]=\sum_{m=0}^{2^{K-1}}\left|s_{m}-\mathrm{E}[X]\right|^{2} \operatorname{Pr}\left(X=s_{m}\right) .
\end{aligned}
$$

Note that $\mathrm{E}[X]$ and $\mathrm{V}[X]$ in (19) are computed for fixed $\left\{\gamma_{k}\right\}$. Similarly to the treatment in Section II-E, assume that $\left\{\gamma_{k}\right\}$ are random and denote by $\mathbb{E}_{\gamma}[\cdot]$ the mathematical expectation over the joint distribution of $\left\{\gamma_{k}\right\}$. We are interested in the impact of signaling scheme $(\mathcal{S}, \mathcal{R})$ on $\mathbb{E}_{\gamma}[\mathrm{V}[X]]$. Now let $X$ be any entry $X[n]$ in $\boldsymbol{X}$. Comparing the definitions in (15) and (19), we can see that

$$
\sigma_{x}^{2}=\mathbb{E}_{\gamma}[\mathrm{V}[X]]
$$

To make a fair comparison of different $(\mathcal{S}, \mathcal{R})$ and following [34], we have two assumptions.

Assumption 5: $(\mathcal{S}, \mathcal{R})$ is unbiased with unit average power: $2^{-K \sum_{m=0}^{2^{K}-1} s_{m}}=0,2^{-K} \sum_{m=0}^{2^{K}-1}\left|s_{m}\right|^{2}=1$.

Assumption 6: $\left\{\gamma_{k}\right\}$ are i.i.d. and their probability density function satisfies the symmetric condition [34]:

$$
p_{\gamma}(\gamma)=p_{\gamma}(-\gamma), \quad \forall \gamma \in\left\{\gamma_{k}\right\} .
$$

We define the variance of a bit after bipolar modulation as

$$
\rho=\mathbb{E}_{\gamma}\left[\mathrm{V}\left[(-1)^{b_{k}}\right]\right]=\mathbb{E}_{\gamma}\left[1-\tanh ^{2}\left(\gamma_{k} / 2\right)\right], \forall k .
$$

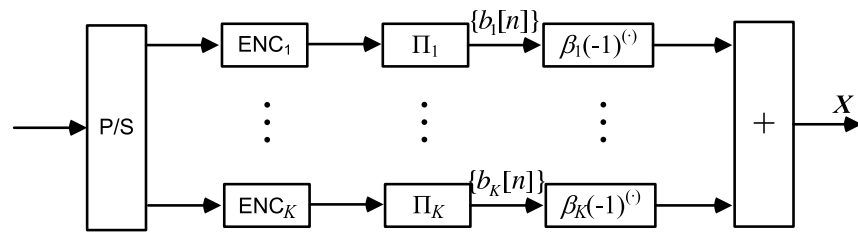

Fig. 3. Transmitter of multi-code SCM.

Here $\rho$ is not a function of $k$ since $\left\{\gamma_{k}\right\}$ are i.i.d.

The following property states that SCM is optimal among all possible signaling schemes $(\mathcal{S}, \mathcal{R})$ in the sense of minimizing $\mathbb{E}_{\gamma}[\mathrm{V}[X]]$. (See [41] for the proof.)

Property 1: Under Assumptions 5 and 6, the minimum symbol variance is given by

$$
\min _{\mathcal{S}, \mathcal{R}} \sigma_{x}^{2}=\min _{\mathcal{S}, \mathcal{R}} \mathbb{E}_{\gamma}[\mathrm{V}[X]]=\rho .
$$

Furthermore, SCM achieves this minimum.

Property 1 indicates that the SCM signaling is an optimal solution to minimize $\sigma_{x}^{2}$ among all possible signaling schemes. Assuming that the function $\sigma_{d}^{2}=\phi\left(\sigma_{x}^{2}\right)$ (see the discussion below (16)) is monotonically increasing, the SCM signaling is also optimal to minimize $\sigma_{d}^{2}$. Note that Property 1 holds for arbitrary constellation sizes, indicating that SCM provides a unified solution to minimize $\sigma_{d}^{2}$ for systems with arbitrary rates.

It is important to note that the optimality of the SCM signaling is with respect to the clipping noise compensation only. SCM is not necessarily optimal in combating the channel noise, which, together with the clipping noise, determines biterror rate (BER). We observed that SCM is particularly attractive when transmission rate is high and/or clipping is deep where the clipping effect becomes dominant, as demonstrated by the numerical results in Section IV.

\section{B. Multi-Code SCM}

A multi-code SCM scheme [25], [28] is illustrated in Fig. 3. For a fixed $k$, we call the set $\left\{(-1)^{b_{k}[n]}\right\}$ as layer- $k$. All the bits in a layer are encoded by a single code and separate encoders are used for different layers. The transmit signal is generated using (17). For the multi-code SCM scheme, the following assumption is approximately true.

\section{Assumption 7:}

(i) the DEC feedback $\left\{\gamma_{k}[n]\right\}$ for the bits in each layer are i.i.d., and

(ii) $\left\{\gamma_{k}[n]\right\}$ may have different distributions for different layers.

We now show that the multi-code SCM scheme can lead to a smaller symbol variance $\mathbb{E}_{\gamma}[\mathrm{V}[X]]$ and thus potentially further improved performance. Denote the symbol variance for the signals in layer- $k$ as (see (22))

$$
\rho_{k}=\mathbb{E}_{\gamma}\left[\mathrm{V}\left[(-1)^{b_{k}[n]}\right]\right]=\mathbb{E}_{\gamma}\left[1-\tanh ^{2}\left(\gamma_{k}[n] / 2\right)\right] .
$$

A smaller $\rho_{k}$ implies that the DEC feedback from layer- $k$ is more reliable. We also define the average of $\left\{\rho_{k}\right\}$ as

$$
\rho \equiv \frac{1}{K} \sum_{k=1}^{K} \rho_{k}
$$


From (17) and (24), the symbol variance for a multi-code SCM scheme is given by

$$
\mathbb{E}_{\gamma}[\mathrm{V}[X]]=\sum_{k=1}^{K}\left|\beta_{k}\right|^{2} \rho_{k} .
$$

When $\left\{\left|\beta_{k}\right|\right\}$ are unequal, without loss of generality, we order $\left\{\left|\beta_{k}\right|\right\}$ as

$$
1 \geq\left|\beta_{1}\right| \geq\left|\beta_{2}\right| \geq \cdots \geq\left|\beta_{K}\right| \geq 0 .
$$

The outputs of a DEC for a layer with a larger $\left|\beta_{k}\right|$ should naturally have a smaller variance. Therefore, $\left\{\rho_{k}\right\}$ will follow the order below.

$$
0 \leq \rho_{1} \leq \rho_{2} \leq \cdots \leq \rho_{K} \leq 1
$$

Recall the average power constraint $\sum_{k=1}^{K}\left|\beta_{k}\right|^{2}=1$. From (25), (27), (28) and Chebyshev's inequality [40],

$$
\mathbb{E}_{\gamma}[\mathrm{V}[X]] \leq \rho .
$$

This can be compared with $\mathbb{E}_{\gamma}[\mathrm{V}[X]]=\rho$ in Property 1 . (Note that Property 1 is based on the assumption that $\left\{\gamma_{k}[n]\right\}$ are all i.i.d., and so $\left\{\rho_{k}\right\}$ are equal, i.e., $\rho_{k}=\rho, \forall k$, but $\left\{\rho_{k}\right\}$ with (29) can be unequal.) From (29), when the overall quality (characterized by $\rho$ ) of the DEC feedback is the same, the multi-code SCM scheme may lead to a smaller symbol variance compared with a BICM-ID scheme.

At the start of iterative process, without decoder feedbacks, we can initialize $\rho=1$ for the single-code scheme in Fig. 1 and $\rho_{k}=1, \forall k$ for the multi-code one in Fig. 3. From (29), as the iterative process proceeds, the symbol variance of the multi-code scheme will always be equal to or less than the single-code one, indicating the former may potentially outperform the latter.

\section{Evolution Analysis}

We now outline a semi-analytical SNR evolution technique to predict the BER of multi-code SCM-based OFDM systems with clipping and iterative soft compensation. It is an extension of the EXIT chart method [34] to multi-code systems. For the OFDM system under consideration, the problem becomes very complicated due to the presence of different types of distortions, including AWGN, frequency-selective fading, clipping distortion, and inter-layer interference related to SCM. In the following, we will show how to characterize these distortions.

From (17), a SCM signal can be expressed as $X[n]=$ $\sum_{k=1}^{K} \beta_{k} X_{k}[n]$, where each $X_{k}[n]=(-1)^{b_{k}[n]} \in\{+1,-1\}$ is a binary phase-shift keying (BPSK) signal. We focus on a particular $X_{k}[n]$ and rewrite the $n$th entry of $\boldsymbol{Z}$ in (7) as

$$
Z[n]=\alpha H[n] \beta_{k} X_{k}[n]+\zeta_{k}[n]
$$

where

$$
\zeta_{k}[n]=\alpha H[n] \sum_{m \neq k} \beta_{m} X_{m}[n]+H[n] D[n]+W[n]
$$

is the distortion component. For notational simplicity, we assume that $\alpha,\left\{\beta_{k}\right\}$ and $H[n]$ in (30) are real numbers. (The results below can be easily generalized when they take complex values.) We treat approximately the distortion term $\zeta_{k}[n]$ as an independent, Gaussian variable. The statistics of $\left\{\zeta_{k}[n]\right\}$ in (30) can be found using the DEC feedback $\left\{\gamma_{m}[n]\right\}$ [35]. Then the extrinsic LLR defined in (10) can be approximated as

$$
\lambda_{k}[n]=\frac{2 \alpha \beta_{k} H[n]}{\operatorname{V}\left[\operatorname{Re}\left(\zeta_{k}[n]\right)\right]}\left(\operatorname{Re}(Z[n])-\mathrm{E}\left[\operatorname{Re}\left(\zeta_{k}[n]\right)\right]\right) .
$$

Substituting (30) into (32), we can rewrite the ESE output into a signal-plus-distortion form as

$$
\begin{aligned}
\lambda_{k}[n]= & \frac{2 \alpha \beta_{k} H[n]}{\mathrm{V}\left[\operatorname{Re}\left(\zeta_{k}[n]\right)\right]} \times \\
& \left(\alpha H[n] \beta_{k} X_{k}[n]+\operatorname{Re}\left(\zeta_{k}[n]\right)-\mathrm{E}\left[\operatorname{Re}\left(\zeta_{k}[n]\right)\right]\right) .
\end{aligned}
$$

In (33), $\operatorname{Re}\left(\zeta_{k}[n]\right)-\mathrm{E}\left[\operatorname{Re}\left(\zeta_{k}[n]\right)\right]$ represents the distortion component with respect to the useful signal $X_{k}[n]$. Its average power can be measured using the symbol variance

$$
\begin{aligned}
\mathbb{E}_{\gamma}\left[\mathrm{V}\left[\operatorname{Re}\left(\zeta_{k}[n]\right)\right]\right] & =\mathbb{E}_{\gamma}\left[\left|\operatorname{Re}\left(\zeta_{k}[n]\right)-\mathrm{E}\left[\operatorname{Re}\left(\zeta_{k}[n]\right)\right]\right|^{2}\right] \\
& =\left|\alpha \beta_{k}\right|^{2}\left(|H[n]|^{2} P_{I, k}+P_{W, k}\right)
\end{aligned}
$$

where $P_{I, k}$ and $P_{W, k}$ are the relative power (normalized by $\left|\alpha \beta_{k}\right|^{2}$ ) of the interference and noise components, respectively, as given below:

$$
\begin{gathered}
P_{I, k}=\sum_{m \neq k} \frac{\left|\beta_{m}\right|^{2}}{\left|\beta_{k}\right|^{2}} \mathbb{E}_{\gamma}\left[\mathrm{V}\left[X_{m}[n]\right]\right]+\frac{\sigma_{d}^{2}}{2\left|\alpha \beta_{k}\right|^{2}}, \\
P_{W, k}=\frac{\sigma_{W}^{2}}{2\left|\alpha \beta_{k}\right|^{2}},
\end{gathered}
$$

where $\sigma_{W}^{2}$ is the average power of the complex channel noise. Then, the SNR for (33) with respect to $X_{k}[n]$ is

$$
\operatorname{snr}_{k}[n]=\frac{\left|\alpha \beta_{k} H[n]\right|^{2}}{\mathbb{E}_{\gamma}\left[\operatorname{V}\left[\operatorname{Re}\left(\zeta_{k}[n]\right)\right]\right]}=\frac{|H[n]|^{2}}{|H[n]|^{2} P_{I, k}+P_{W, k}} .
$$

The following assumption can greatly simplify the analysis problem.

\section{Assumption 8:}

(i) The inputs $\left\{\lambda_{k}[n]\right\}$ to the DEC at different time are independent. Similarly, the feedbacks $\left\{\gamma_{k}[n]\right\}$ from the DEC at different time are also independent.

(ii) The input sequence of the DEC is characterized by $\left\{\operatorname{snr}_{k}[n]\right\}$ in (37).

(iii) The distribution of $\{H[n]\}$ is given.

Note that (i) is true when infinite-length random interleavers are assumed, and (ii) holds when the Gaussian assumption is applied to $\left\{\zeta_{k}[n]\right\}$. In (iii), a typical case is that $\{H[n]\}$ follows Rayleigh distribution. Based on Assumption 8 and from (37), the pair $\left(P_{I, k}, P_{W, k}\right)$ fully determines the DEC performance. In the iterative decoding process, $P_{W, k}$ is a constant but $P_{I, k}$ may decrease as the iteration proceeds. We discuss below how to track $P_{I, k}$.

From our earlier definitions, $\rho_{k}=\mathbb{E}_{\gamma}\left[\mathrm{V}\left[X_{k}[n]\right]\right], k=$ $1,2, \cdots, K$. Now, $\sigma_{x}^{2}=\sum_{k=1}^{K}\left|\beta_{k}\right|^{2} \rho_{k}$. Therefore, from (16), $\sigma_{d}^{2}$ can be found from $\left\{\rho_{k}\right\}$ as

$$
\sigma_{d}^{2}=\phi\left(\sum_{k=1}^{K}\left|\beta_{k}\right|^{2} \rho_{k}\right),
$$




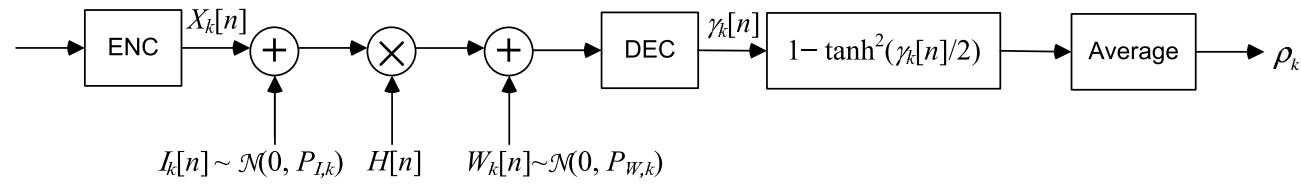

Fig. 4. Block diagram of the Monte Carlo simulation for the global variance $\rho_{k}$.

where $\phi(\cdot)$ is defined in (16). From (35) and (38), $P_{I, k}$ is fully determined by $\left\{\rho_{k}\right\}$. On the other hand, since $\rho_{k}$ is the variance of the DEC feedback of the $k$ th layer, it is a function of $P_{I, k}$ and $P_{W, k}$ that characterize the inputs to the DEC of the $k$ th layer. We write this function as

$$
\rho_{k}=f\left(P_{I, k}, P_{W, k}\right) .
$$

Note that here the decoder is characterized by a bivariate function $f(\cdot)$, differing from the treatments with univariate $f(\cdot)$ in [35], [36]. This is because, for OFDM over frequency-selective channels, the clipping noise plus inter-layer interference and AWGN (characterized by $P_{I, k}$ and $P_{W, k}$, respectively) have different impact on $\left\{\operatorname{snr}_{k}[n]\right\}$ in (37).

In general, $f(\cdot)$ cannot be expressed in a closed form, but it can be characterized by a look-up table created by the Monte Carlo simulation. The block diagram of the simulation is depicted in Fig. 4, where we have used an equivalent channel model

$$
Z_{k}[n]=H[n]\left(X_{k}[n]+I_{k}[n]\right)+W_{k}[n],
$$

where $X_{k}[n] \in\{+1,-1\}$ is the coded BPSK signal, $I_{k}[n] \sim$ $\mathcal{N}\left(0, P_{I, k}\right)$ and $W_{k}[n] \sim \mathcal{N}\left(0, P_{W, k}\right)$, respectively, represent the (normalized) interference and channel noise. As shown in Fig. 4, $\rho_{k}$ is estimated using the average of the DEC outputs. Similarly, the BER performance of the DEC can be characterized by a function as

$$
\mathrm{BER}_{k}=g\left(P_{I, k}, P_{W, k}\right) .
$$

To summarize, we can characterize the iterative decoding process using the following procedure. (We assume $Q$ iterations and denote by $(\cdot)^{(q)}$ the $q$ th iteration.)

\section{SNR Evolution:}

(i) Initialization: Set $q=1$ and $\rho_{k}^{(q)}=1, P_{W, k}=\frac{\sigma_{W}^{2}}{2\left|\alpha \beta_{k}\right|^{2}}$, $k=1,2, \cdots, K$.

(ii) For the $q$ th iteration:

Find the normalized interference power for the ESE:

$$
\begin{gathered}
P_{I, k}^{(q)}=\sum_{m \neq k} \frac{\left|\beta_{m}\right|^{2}}{\left|\beta_{k}\right|^{2}} \rho_{m}^{(q)}+\frac{\phi\left(\sum_{m=1}^{K}\left|\beta_{m}\right|^{2} \rho_{m}^{(q)}\right)}{2\left|\alpha \beta_{k}\right|^{2}}, \\
k=1,2, \cdots, K .
\end{gathered}
$$

Find the output variance of the DEC:

$$
\rho_{k}^{(q+1)}=f\left(P_{I, k}^{(q)}, P_{W, k}\right), \quad k=1,2, \cdots, K .
$$

(iii) Recursion: If $q<Q$, set $q \leftarrow q+1$ and go to (ii); otherwise, go to (iv).

(iv) Find the BER for each layer:

$$
\mathrm{BER}_{k}=g\left(P_{I, k}^{(q)}, P_{W, k}\right), k=1,2, \cdots, K .
$$

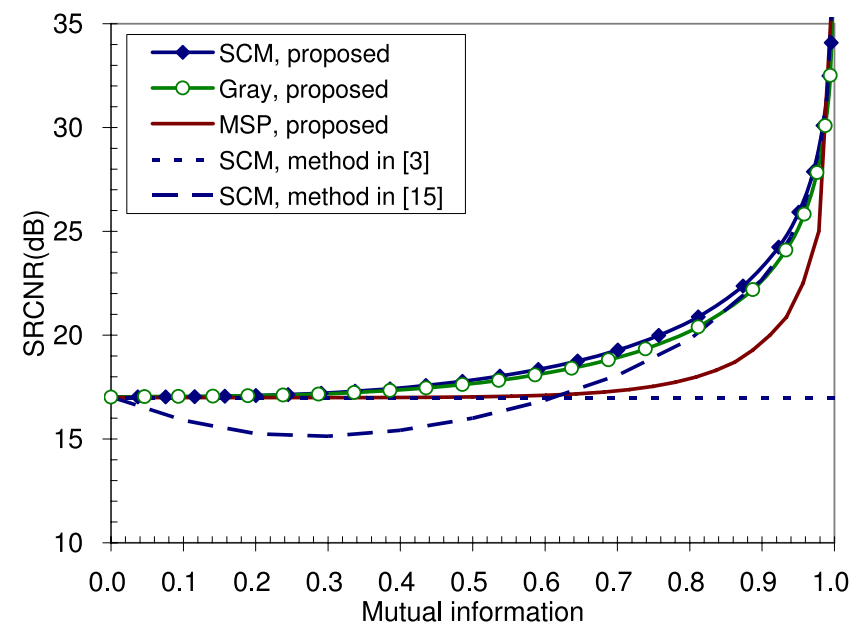

Fig. 5. Impact of detection methods and signaling schemes on SRCNR. $L=4, N=256$, and $\mathrm{CR}=0 \mathrm{~dB}$.

With the above technique, only the binary-input system in Fig. 4 needs to be simulated. Then using the stored lookup tables to characterize (16), (39) and (41), one can predict the performance of multi-code SCM with arbitrary weighting factors $\left\{\beta_{k}\right\}$ and channel SNR. This is more convenient than the EXIT chart method in BICM-ID where Monte Carlo simulations of a multi-ary system have to be performed whenever the signaling scheme or channel SNR is changed. The SNR evolution technique can also be used for optimizing the weighting factors $\left\{\beta_{k}\right\}$. The related optimization technique is beyond the scope of this paper. Interested readers may refer to [25], [35] for related discussions.

\section{Numerical Results}

This section presents numerical results. We take OFDM systems based on the BICM-ID [19], [20] and multi-code SCM schemes [24]-[28] as examples. For SCM, we assume even $K$ and $\beta_{k-1}=i \beta_{k}$ with $i=\sqrt{-1}$ and $\beta_{k-1}$ being a real number for $k=2,4, \cdots, K$. The APP and Gaussian approximation (GA) [28] demapping methods are applied to the BICM-ID and multi-code SCM schemes, respectively. The number of subcarriers $N=256$ and the oversampling factor $L=4$.

1) SRCNR: We first show the effectiveness of the proposed soft compensation method by comparing it with the background work in [3] and [15], where the clipping noise $\boldsymbol{d}$ modeled in (4) are treated as an additive Gaussian noise and estimated as $g(\mathrm{E}[\boldsymbol{x}])-\alpha \mathrm{E}[\boldsymbol{x}]$, respectively. (See also Section II-D.) Define the signal to residual clipping noise ratio (SRCNR) from (7) and (8) as

$$
\operatorname{SRCNR}=\frac{|\alpha|^{2} \mathrm{E}\left[|X[n]|^{2}\right]}{\sigma_{d}^{2}} .
$$




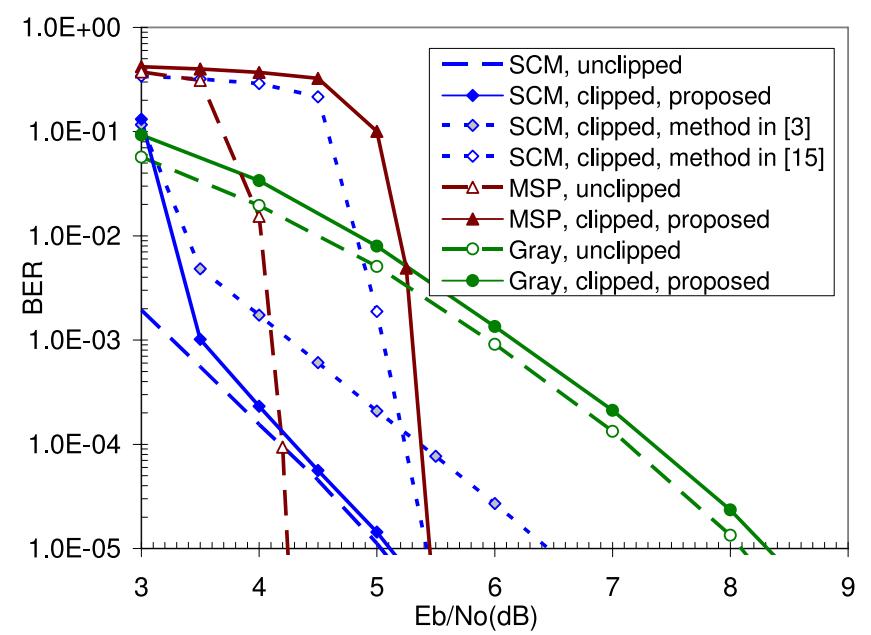

Fig. 6. Comparison of different detection methods and signaling schemes for clipped BICM-ID-OFDM schemes over AWGN channels. For SCM, $K=4,\left\{\left|\beta_{k}\right|\right\}=\{1 \times 4\} . L=4, N=256, \mathrm{CR}=0 \mathrm{~dB}$. The rate- $1 / 2$ convolutional code $(23,35)_{8}$ is used. The system rate is $R=2$ bits/symbol. The frame length is 4096 . (Each frame consists of 16 OFDM symbols.) The number of iterations is 12 .

Here, the residual clipping noise power $\sigma_{d}^{2}$ defined in (14) is measured using Monte-Carlo simulation and applying the different methods to the SC module in Fig. 1. We assume i.i.d., consistent Gaussian DEC feedbacks $\left\{\gamma_{k}[n]\right\}$, which is reasonable in BICM-ID. Then, the mutual information $\left(I_{\gamma}\right)$ between $\left\{\gamma_{k}[n]\right\}$ and the coded bits $\left\{b_{k}[n]\right\}$ can characterize the distribution of $\left\{\gamma_{k}[n]\right\}$.

The SRCNR achieved by different methods for an SCM signaling with $K=4$ are compared in Fig. 5. The proposed method consistently outperforms the alternatives in [3] and [15]. This is because it estimates $\boldsymbol{d}$ as the conditional mean $\overline{\boldsymbol{d}}=\mathrm{E}[g(\boldsymbol{x})-\alpha \boldsymbol{x}]$, which is optimal when the a priori distribution of $\boldsymbol{x}$ is available. The SRCNR with the method in [15] drops as $I_{\gamma}$ increases from 0 to 0.25 and is even worse than the method in [3] where $\boldsymbol{d}$ is treated as a Gaussian noise without mitigation. This indicates that the method in [15] does not necessarily yield better estimate of $\boldsymbol{d}$ if the estimates of $\boldsymbol{x}$ are improved. By contrast, Fig. 2 shows that the proposed method does lead to better estimates of $\boldsymbol{d}$ with better estimates of $\boldsymbol{x}$.

We also compare the SCM signaling and the 16-QAM signaling schemes with Gray and MSP mappings [19] for the proposed method. From Fig. 5, the SCM signaling is clearly the best choice. This confirms the discussion in Section III that the SCM signaling is optimal for minimizing the residual clipping noise power $\sigma_{d}^{2}$. Note that the Gray signaling can also achieve good SRCNR according to Fig. 5.

2) BER in AWGN Channels: Fig. 6 compares BERs of a BICM-ID-OFDM system with the different detection methods and signaling schemes in Fig. 5, where we have defined $E_{b} / N_{0}=10 \log _{10}\left(\mathrm{E}\left[\|\boldsymbol{F} g(\boldsymbol{x})\|^{2}\right] /\left(N R \sigma_{W}^{2}\right)\right)$ in decibel with $R$ being the system rate. The proposed soft compensation method based on (12) and (13) significantly outperforms the other two approaches. It has roughly the same computational complexity as the alternatives (except the extra memory required to store the look-up tables). Clearly, SCM is more

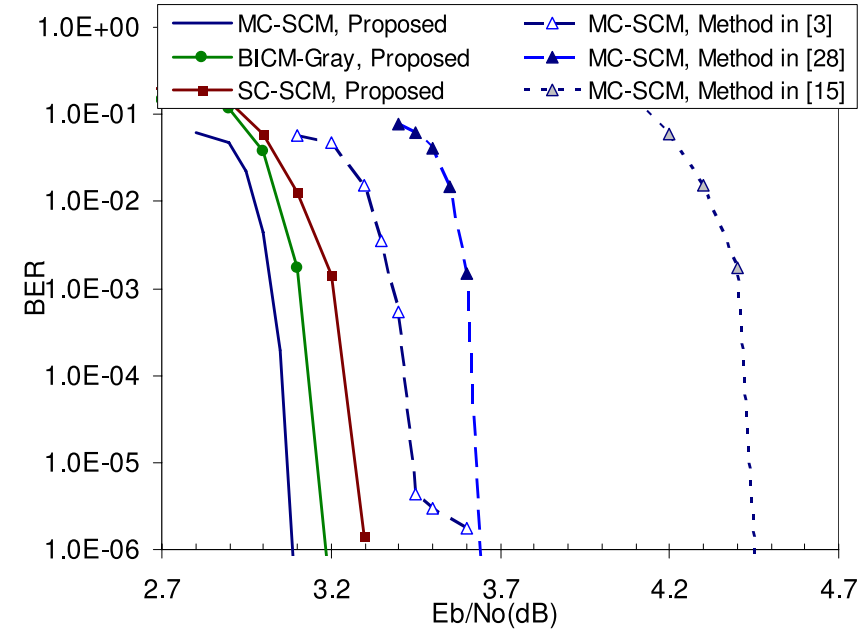

Fig. 7. Comparisons of LDPC-coded BICM-ID-OFDM, SC-SCM-OFDM and MC-SCM-OFDM with clipping over AWGN channels. $L=4, N=256$, $\mathrm{CR}=0 \mathrm{~dB}$. The frame length is 32768. $R=2$. For BICM-Gray and SCSCM, the number of outer iterations (between the ESE and DEC) is 1000 and the number of inner iterations (of LDPC decoding) is 1. For MC-SCM, the number of outer iterations is 10 and the number of inner iterations is 20 . For all the SCM schemes, $\left\{\left|\beta_{k}\right|\right\}=\{1 \times 2,1.5 \times 2\}$. The constellation of BICM-Gray is 16-QAM.

robust against the clipping effect than its alternatives, which is in line with the SRCNR results in Fig. 5.

Fig. 7 compares BICM-ID with the single- and multi-code SCM schemes with a rate- $1 / 2$ LDPC code and $K=4$. Following [43], the performance with LDPC codes can be improved by optimizing the node degree distributions. The best results (for $\mathrm{CR}=0 \mathrm{~dB}$ with the proposed soft compensation method) known to us are applied in Fig. 7:

- Single-code SCM (SC-SCM): the degree profile of variable nodes $\lambda(x)=0.2394 x^{2}+0.1824 x^{3}+0.0682 x^{5}+$ $0.0175 x^{7}+0.1907 x^{8}+0.3018 x^{30}$, and the degree profile of check nodes $\rho(x)=0.3 x^{8}+0.7 x^{9}$.

- BICM-Gray: $\lambda(x)=0.3965 x^{2}+0.2272 x^{3}+0.0583 x^{10}+$ $0.0842 x^{11}+0.2338 x^{30}$ and $\rho(x)=0.2 x^{6}+0.8 x^{7}$.

- Multi-code SCM (MC-SCM): $\lambda(x)=0.25 x^{2}+$ $0.1854 x^{3}+0.0031 x^{4}+0.1499 x^{5}+0.1616 x^{8}+$ $0.0055 x^{10}+0.0423 x^{20}+0.2022 x^{21}$ and $\rho(x)=x^{8}$.

From Fig. 7, the MC-SCM scheme yields the best performance (within $1.35 \mathrm{~dB}$ from the Shannon limit) with the proposed soft compensation method. This scheme also has lower complexity than the alternatives in Fig. 7 as a smaller number of iterations are applied. BICM-Gray outperforms the SC-SCM slightly by $0.12 \mathrm{~dB}$, which indicates that the BER performance is not determined by Property 1 alone. (Recall that, from Fig. 5, the difference in SRCNR between the SCM and Gray signaling schemes is very small.) From Fig. 7, the proposed soft compensation technique provides noticeable performance improvement compared with those proposed in [3], [15] and [28]. This confirms our discussions in Section II-D.

3) BER in Fading Channels: We also consider Rayleigh fading channels. The multi-code SCM and BICM-ID schemes at rate $R=4$ bits/symbol are compared. For SCM, the component code is a concatenation of the rate- $1 / 2$ convolutional code $(23,35)_{8}$ with a length-4 repetition code [35]; $K=32$. For BICM-ID, the 64-QAM with set-partitioning (SP) mapping 


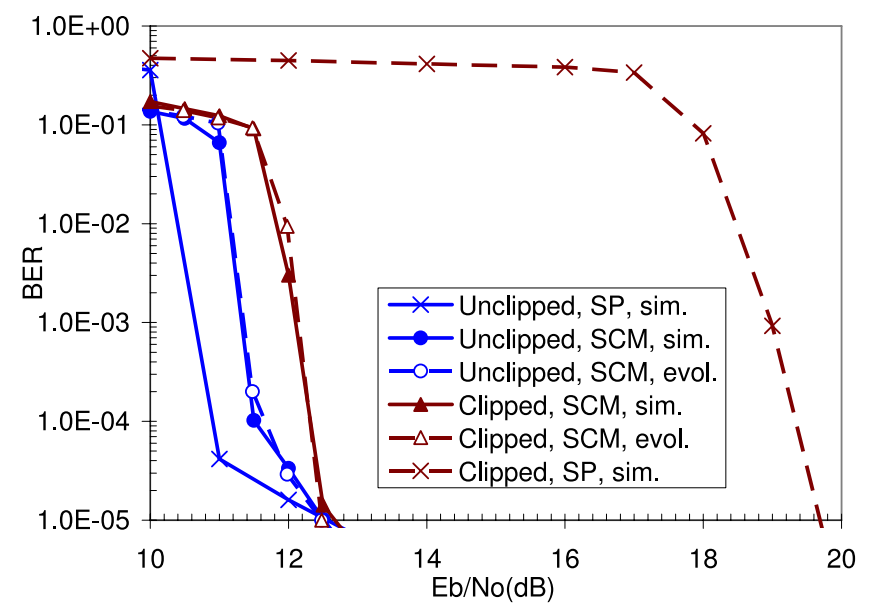

Fig. 8. Comparison of convolutional-coded multi-code SCM-OFDM and BICM-ID-OFDM with clipping and soft compensation over fully interleaved Rayleigh fading channels. $L=4, N=256$, and $\mathrm{CR}=2 \mathrm{~dB}$. The frame length is 16384 . $R=4$. The number of iterations is 12 . The weighting factors of SCM are given by $\left\{\left|\beta_{k}\right|\right\}=\{1 \times 12,1.58 \times 4,2.10 \times 6,2.49 \times 2,2.73 \times$ $2,3.58 \times 4,3.93 \times 2\}$.

and $K=6$ is used [38] and the above convolutional code is punctured to rate $2 / 3$ to achieve $R=4$. The APP and GA [28] demapping methods at complexities of $\mathcal{O}\left(2^{K}\right)$ and $\mathcal{O}(K)$ are applied to the BICM-ID and SCM schemes, respectively. As $K=6$ for the BICM-ID and $K=32$ for the SCM, the schemes compared here have comparable demapping complexities. The decoding complexities of the component codes are also comparable [35].

From Fig. 8, without clipping, the BICM-ID scheme with SP signaling and the SCM scheme have similar performance. However, when clipping is used, SCM significantly outperforms BICM-ID. In this case, the iterative compensation is not very effective for the BICM-ID scheme with the SP signaling; hence, its performance is dramatically degraded by the clipping effect. Soft compensation still works well with the SCM signaling in this case.

Fig. 8 also compares the simulation results and the BER predicted by the SNR evolution technique outlined in Section III-C. From Fig. 8, they agree well in both the clipped and unclipped cases. This clearly demonstrates the effectiveness of the SNR evolution technique.

\section{CONCLUSION}

Clipping can alleviate the high PAPR problem in OFDM systems but causes serious performance degradation when not treated properly. In this paper, we propose an iterative soft compensation method to mitigate the clipping effect, which can outperform conventional approaches. We analyze the impact of signaling schemes on performance and show the optimality of SCM signaling for clipping noise compensation. We also show that the performance can be improved by using mutli-code SCM schemes whose performance can be predicted and optimized based on a fast evolution technique. Both the analytical and numerical results show that the SCM scheme with the proposed soft compensation method can outperform other alternatives, especially when clipping is severe and/or transmission rate is high.

\section{ACKNOWLEDGEMENT}

The authors would like to thank the three anonymous reviewers and the editor for their valuable comments and suggestions that helped improve the quality of this paper.

\section{REFERENCES}

[1] S. H. Han and J. H. Lee, "An overview of peak-to-average power ratio reduction techniques for multicarrier transmission," IEEE Wireless Commun., vol. 12, no. 2, pp. 56-65, Apr. 2005.

[2] X. Li and L. J. Cimini, "Effects of clipping and filtering on the performance of OFDM," IEEE Commun. Lett., vol. 2, no. 5, pp. 131133, May 1998.

[3] H. Ochiai and H. Imai, "Performance analysis of deliberately clipped OFDM signals," IEEE Trans. Commun., vol. 50, no. 1, pp. 89-101, Jan. 2002.

[4] A. R. S. Bahai, M. Singh, A. J. Goldsmith, and B. R. Saltzberg, "A new approach for evaluating clipping distortion in multicarrier," IEEE J. Sel. Areas Commun., vol. 20, no. 5, pp. 1037-1046, May 2002.

[5] R. Dinis and A. Gusmao, "On the performance evaluation of OFDM transmission using clipping techniques, “ in Proc. IEEE VTC'99 (Fall), Amsterdam, Sept. 1999.

[6] J. Armstrong, "Peak-to-average power reduction for OFDM by repeated clipping and frequency domain filtering," Electron. Lett., vol. 38, no. 5, pp. 246-247, Feb. 2002.

[7] X. Huang, J. Lu, J. Lu, K. B. Letaief, and J. Gu, "Companding transform for reduction in peak-to-average power ratio of OFDM signals," IEEE Trans. Wireless Commun., vol. 3, no. 6, pp. 2030-2039, Nov. 2004.

[8] P. Banelli, G. Leus, and G. B. Giannakis, "Bayesian estimation of clipped Gaussian process with application to OFDM," in Proc. EUSIPCO'02, Sep. 2002.

[9] D. Declercq, "Recovering clipped OFDM symbols with Bayesian inference," in Proc. ICASSP'O0, vol. 1, pp. 157-160, June 2000.

[10] D. Kim and G. L. Stüber, "Clipping noise mitigation for OFDM by decision-aided reconstruction," IEEE Commun. Lett., vol. 3, no. 1, pp. 4-6, Jan. 1999.

[11] M. Colas, G. Gelle, and D. Declercq, "Turbo decision aided receivers for clipping noise mitigation in coded OFDM," EURASIP J. Wireless Commun. Network., vol. 2008, pp. 1-10, Feb. 2008.

[12] H. Chen and A. M. Haimovich, "Iterative estimation and cancellation of clipping noise for OFDM signals," IEEE Commun. Lett., vol. 7, no. 7, pp. 305-307, July 2003.

[13] H. Nikopour, A. K. Khandani, and S. H. Jamali, "Turbo-coded OFDM transmission over a nonlinear channel," IEEE Trans. Veh. Technol., vol. 54, no. 4, pp. 1361-1371, July 2005.

[14] J. Tellado, L. M. C. Hoo, and J. M. Cioffi, "Maximum-likelihood detection of nonlinearity distorted multicarrier symbols by iterative decoding," IEEE Trans. Commun., vol. 51, no. 2, pp. 218-228, Feb. 2003.

[15] W. Rave, P. Zillmann, and G. Fettweis, "Iterative correction and decoding of OFDM signals affected by clipping," in Proc. Int. Workshop Multicarrier Spread Spectrum (MC-SS'05), Germany, Sep. 2005.

[16] F. Peng and W. E. Ryan, "On the capacity of clipped OFDM channels," in Proc. IEEE Int. Symp. Inf. Theory, pp. 1866-1870, Seattle, WA, July 2006.

[17] F. Peng and W. E. Ryan, "MLSD bounds and receiver design for clipped OFDM channels," IEEE Trans. Wireless Commun., vol. 7, no. 9, pp. 3568-3578, Sep. 2008.

[18] H. Ochiai, "Performance of optimal and suboptimal detection for uncoded OFDM system with deliberate clipping and filtering," in Proc. IEEE GlobeCom 2003, pp. 1618-1622, San Francisco, CA, Dec. 2003.

[19] A. Chindapol and J. A. Ritcey, "Design, analysis, and performance evaluation for BICM-ID with square QAM constellations in Rayleigh fading channels," IEEE J. Sel. Areas Commun., vol. 19. no. 5, pp. 944957, May 2001.

[20] J. Tan and G. Stüber, "Analysis and design of symbol mappers for iteratively decoded BICM," IEEE Trans. Wireless Commun., vol. 4, no. 2, pp. 662-672, Mar. 2005.

[21] A. Nilsson and T. M. Aulin, "Bit interleaved coded modulation with multi-labeled signal mapping," in Proc. IEEE ISIT'2007, Nice, France, June 2007.

[22] F. Schreckenbach, N. Gortz, J. Hagenauer, and G. Bauch, "Optimized symbol mappings for bit-interleaved coded modulation with iterative decoding," in Proc. IEEE Globecom 2003, pp. 3316-3320, San Francisco, CA, Dec. 2003. 
[23] U. Wachsmann, R. F. H. Fischer, and J. B. Huber, "Multilevel codes: theoretical concepts and practical design rules," IEEE Trans. Inf. Theory, vol. 45, no. 5, pp. 1361-1391, July 1999.

[24] L. Duan, B. Rimoldi, and R. Urbanke, "Approaching the AWGN channel capacity without active shaping," in Proc. ISIT'97, Ulm, Germany, p. 374, July 1997.

[25] X. Ma and Li Ping, "Coded modulation using superimposed binary codes," IEEE Trans. Inf. Theory, vol. 50, no. 12, pp. 3331-3343, Dec. 2004.

[26] T. W. Sun, R. D. Wesel, M. R. Shane, and K. Jarett, "Superposition turbo TCM for multirate broadcast," IEEE Trans. Commun., vol. 52, no. 3, pp. 368-371, Mar. 2004.

[27] N. H. Tran, H. H. Nguyen, and Tho. Le-Ngoc, "Performance of BICMID with signal space diversity," IEEE Trans. Wireless Commun., vol. 6, no. 5, pp. 1732-1742, May 2007.

[28] J. Tong, Li Ping, and X. Ma, "Superposition coded modulation with peak-power-limitation," IEEE Trans. Inf. Theory, vol. 55, no. 6, pp. 2562-2576, June 2009.

[29] P. A. Hoeher, H. Schoeneich, and J. Ch. Fricke, "Multi-layer interleavedivision multiple access: theory and practice," European Trans. Telecommun., vol. 19, no. 5, Aug. 2008.

[30] J. Tong and Li Ping, "Performance analysis of superposition coded modulation," Phys. Commun., vol. 3, no. 3, pp. 147-155, Sep. 2010.

[31] S. M. Kay, Fundamentals of Signal Processing: Estimation Theory, Prentice Hall International Editions, 1993.

[32] R. Price, "A useful theorem for nonlinear devices having Gaussian inputs," IRE Trans. Inf. Theory, vol. IT-4, pp. 69-72, June 1958.

[33] S. ten Brink, J. Speidel, and R. Yan, "Iterative demapping and decoding for multilevel modulation," in Proc. IEEE Globecom 1998, pp. 579-584, Sydney, Australia, Nov. 1998.

[34] S. ten Brink, "Convergence behavior of iteratively decoded parallel concatenated codes," IEEE Trans. Commun., vol. 49, no. 10, pp. 17271737 , Oct. 2001

[35] L. H. Liu, J. Tong, and Li Ping, "Analysis and optimization of CDMA systems with chip-level interleavers," IEEE J. Sel. Areas Commun., vol. 24, no. 1, pp. 141-150, Jan. 2006.

[36] X. Yuan, Q. Guo, X. Wang, and Li Ping, "Evolution analysis of lowcost iterative equalization in coded linear systems with cyclic prefixes," IEEE J. Sel. Areas Commun., vol. 26, no. 2, pp. 301-310, Feb. 2008.

[37] G. Caire, G. Taricco, and E. Biglieri, "Bit-interleaved coded modulation," IEEE Trans. Inf. Theory, vol. 44, no. 3, pp. 927-946, May 1998.

[38] S. Pfletschinger and F. Sanzi, "Error floor removal for bit-interleaved coded modulation with iterative detection," IEEE Trans. Wireless Commun., vol. 5, no. 11 pp. 3174-3181, Nov. 2006.

[39] J. G. Proakis, Digital Communications, 4th edition. New York: McGrawHill, 2000.

[40] G. H. Hardy, J. E. Littlewood, and G. Polya, Inequalities, 2nd edition. Cambridge, U.K.: Cambridge Univ. Press, 1959.

[41] Li Ping, J. Tong, X. Yuan, and Q. Guo, "Superposition coded modulation and iterative linear MMSE detection," IEEE J. Sel. Areas Commun., vol. 27. no. 6, pp. 995-1004, Aug. 2009.

[42] R. Y. S. Tee, S. X. Ng, and L. Hanzo, "Three-dimensional EXIT chart analysis of iterative detection aided coded modulation schemes," in Proc. IEEE VTC'06 Spring, pp. 2494-2498, Melbourne, Australia.

[43] S. ten Brink, G. Kramer, and A. Ashikhmin, "Design of low-density parity-check codes for modulation and detection," IEEE Trans. Commun., vol. 52, no. 4, pp. 670-678, Apr. 2004.

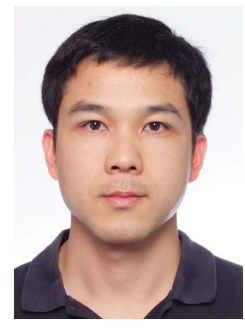

Jun Tong received the B.E. degree in information engineering and the M.E. degree in signal and information processing from the University of Electronic Science and Technology of China, in 2001 and 2004 respectively, and the $\mathrm{Ph}$. D. degree in electronic engineering from City University of Hong Kong in 2009. He is now with the School of Electrical Engineering and Computer Science, The University of Newcastle, NSW, Australia. His research interests are in the areas of signal processing and communication techniques.

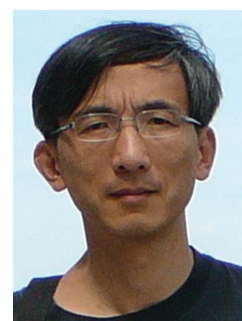

Li Ping (S'87-M'91-SM'06-F'09) received his $\mathrm{Ph} . \mathrm{D}$. degree at Glasgow University in 1990. He lectured at Department of Electronic Engineering, Melbourne University, from 1990 to 1992, and worked as a member of research staff at Telecom Australia Research Laboratories from 1993 to 1995. $\mathrm{He}$ has been with the Department of Electronic Engineering, City University of Hong Kong, since January 1996, where he is now a chair professor. His research interests are communications systems and coding theory.

Dr. Li Ping received a British Telecom-Royal Society Fellowship in 1986, the IEE J J Thomson premium in 1993 and the Croucher Foundation award in 2005 .

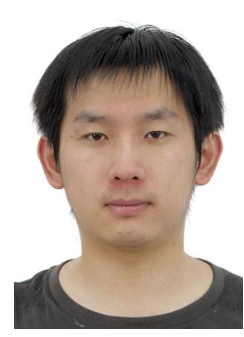

Zhonghao Zhang received the B.Eng. degree and the M.Sc. degree from the University of Electronic Science and Technology of China in 2005 and 2008, respectively, both in electronic engineering. $\mathrm{He}$ is currently pursuing the Ph.D. degree at City University of Hong Kong. His research interests span the areas of digital wireless communications and net information theory.

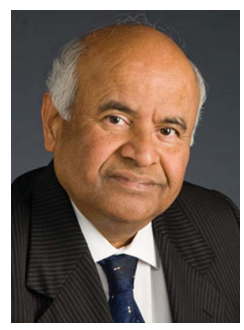

Vijay Bhargava (S'70-M'74-SM'82-F'92), an IEEE volunteer for three decades, is Professor in the Department of Electrical and Computer Engineering at the University of British Columbia in Vancouver, where he served as Department Head during 20032008. Previously he was with the University of Victoria (1984-2003) and Concordia University (197684). He received his Ph.D. from Queen's University. As a distinguished speaker for the IEEE Communications Society, the IEEE Information Theory Society and as a senior level IEEE volunteer, he has lectured in 66 countries and assisted IEEE Presidents in negotiating sister society agreements in India, Japan and Russia. He has rudimentary knowledge of several languages and is an eager student of different cultures and societies.

Vijay served as the Founder and President of Binary Communications Inc. (1983-2000). He has provided consulting services to several companies and government agencies. He is a co-author (with D. Haccoun, R. Matyas, and P. Nuspl) of Digital Communications by Satellite (New York: Wiley: 1981), which was translated into Chinese and Japanese. He is a co-editor (with S. Wicker) of Reed Solomon Codes and their Applications (IEEE Press: 1994), a co-editor (with V. Poor, V. Tarokh, and S. Yoon) of Communications, Information and Network Security (Kluwer: 2003), a co-editor (with E. Hossain) of Cognitive Wireless Communication Networks (Springer: 2007), and a co-editor (with E. Hossain and D. I. Kim) of Cooperative Wireless Communications Networks, a forthcoming Cambridge University Press Publication.

Vijay has served on the Board of Governors of the IEEE Information Theory Society and the IEEE Communications Society. He has held important positions in these societies and has organized conferences such as ISIT'95, ICC'99, VTC 2002 Fall. He has served as an Editor of the IEEE TRANSACTIONS ON COMMUNICATIONS. He played a major role in the creation of the IEEE Communications and Networking Conference (WCNC) and IEEE TRANSACTIONS ON WIRELESS COMMUNICATIONS, for which he served as the editor-in-chief during 2007, 2008 and 2009. In 2010, he was appointed for a two year term as the IEEE Communications Society Director of Journals. He is a past President of the IEEE Information Theory Society. Vijay Bhargava is the IEEE Communications Society's President-Elect. 